\title{
Primary esophageal and gastro-esophageal junction cancer xenograft models: clinicopathological features and engraftment
}

Lorin Dodbiba ${ }^{1}$, Jennifer Teichman ${ }^{1}$, Andrew Fleet ${ }^{2}$, Henry Thai ${ }^{2}$, Bin Sun ${ }^{2,3}$, Devang Panchal ${ }^{2,4}$, Devalben Patel ${ }^{2}$, Alvina Tse ${ }^{2}$, Zhuo Chen², Olusola O Faluyi ${ }^{4}$, Daniel J Renouf ${ }^{4}$, Hala Girgis ${ }^{5}$, Bizhan Bandarchi ${ }^{2}$, Joerg Schwock ${ }^{5}$, Wei $\mathrm{Xu}^{6}$, Robert G Bristow ${ }^{1,2,4}$, Ming-Sound Tsao ${ }^{2,4}$, Gail E Darling ${ }^{7}$, Laurie E Ailles ${ }^{1,2}$, Hala El-Zimaity ${ }^{5}$ and Geoffrey Liu ${ }^{1,2,4,8}$

There are very few xenograft models available for the study of esophageal (E) and gastro-esophageal junction (GEJ) cancer. Using a NOD/SCID model, we implanted 90 primary E and GEJ tumors resected from patients and six endoscopic biopsy specimens. Of 69 resected tumors with histologically confirmed viable adenocarcinoma or squamous cell carcinoma, 22 (32\%) was engrafted. One of 11 tumors, considered to have had a complete pathological response to neo-adjuvant chemo-radiation, also engrafted. Of the 23 patients whose tumors were engrafted, $65 \%$ were male; $30 \%$ were early stage while $70 \%$ were late stage; $22 \%$ received neo-adjuvant chemo-radiation; $61 \%$ were GEJ cancers. Engraftment occurred in 18/54 (33\%) adenocarcinomas and 5/16 (31\%) squamous cell carcinomas. Small endoscopic biopsy tissue had a 50\% (3/6) engraftment rate. Of the factors analyzed, pretreatment with chemo-radiation and well/moderate differentiation showed significantly lower correlation with engraftment $(P<0.05)$. In the subset of patients who did not receive neo-adjuvant chemo-radiation, 18/41 (44\%) engrafted compared with those with pretreatment where $5 / 29(17 \%, P=0.02)$ engrafted. Primary xenograft lines may be continued through 4-12 passages. Xenografts maintained similar histology and morphological characteristics with only minor variations even after multiple passaging in most instances.

Laboratory Investigation (2013) 93, 397-407; doi:10.1038/labinvest.2013.8; published online 11 February 2013

KEYWORDS: adenocarcinoma; clinicopathological features; engraftment; esophageal cancer; GE-Junction; primary xenograft; squamous cell carcinoma

Experimental models of cancer have been crucial in the understanding of tumor biology and in the development of new therapies. In esophageal (E) and gastro-esophageal junction (GEJ) cancer, there has been a steep rise in incidence in developed countries while the 5-year-survival remains as low as $19 \% .{ }^{1}$ It has been challenging for preclinical models of E/GEJ cancer to reproduce tumor characteristics and heterogeneity. Traditionally, E/GEJ cancer cell lines have been used either in vitro or injected into immunocompromised mice in order to study the effects of new therapeutics. In general, this method has been successful in interrogating large numbers of potential therapies in many types of cancers. ${ }^{2,3}$ However, cancer cell lines may lack some of the molecular heterogeneity seen in primary samples and may acquire in vitro mutations, which can alter both genomic and phenotypic factors compared with the patient's original tumor, ${ }^{4-6}$ although other studies dispute these findings. ${ }^{7}$ In the case of E/GEJ cancer, the popular commercial cell lines SEG-1, BIC-1 and SK-GT5 were recently discovered to be contaminated. ${ }^{8}$ Thus, there is a need to develop better preclinical models of E/GEJ cancer that may complement in vitro cell line models.

Primary tumor xenografts (PTXGs) show promising signs of being an alternative preclinical model of disease. PTXGs

\footnotetext{
${ }^{1}$ Department of Medical Biophysics, University of Toronto, Toronto, ON, Canada; ${ }^{2}$ Ontario Cancer Institute, Toronto, ON, Canada; ${ }^{3}$ Department of Mathematics and Statistics, York University, Toronto, ON, Canada; ${ }^{4}$ University Health Network, Princess Margaret Hospital, Toronto, ON, Canada; ${ }^{5}$ Department of Anatomical Pathology, Toronto General Hospital, Toronto, ON, Canada; ${ }^{6}$ Department of Biostatistics, Princess Margaret Hospital, Toronto, ON, Canada; ${ }^{7}$ Division of Thoracic Surgery, Toronto General Hospital, Toronto, ON, Canada and ${ }^{8}$ Division of Epidemiology, Dalla Lana School of Public Health, Toronto, ON, Canada

Correspondence: Dr H El-Zimaity, MD, MSc, Toronto General Hospital, 200 Elizabeth Street, Rm 11E210, Toronto, Ontario M5G 2C4, Canada.

E-mail: hala.el-zimaity@uhn.ca or Dr G Liu, MD, MSc, FRCPC Princess Margaret Hospital 610 University Ave, Rm 7-124, Toronto M5T 2M9, Ontario, Canada.

E-mail: geoffrey.liu@uhn.ca

Received 2 October 2012; revised 9 January 2013; accepted 10 January 2013
} 
are created by implanting tumor fragments from a patient into immunocompromised mice and using the resultant tumor for tissue expansion and subsequent experimentation. Similar models of lung, ${ }^{9}$ breast, ${ }^{10}$ pancreatic, ${ }^{11}$ colon, ${ }^{12}$ head and neck $^{13}$ and various other gastrointestinal and female genital cancers ${ }^{14}$ have been shown to recapitulate the original tumor by having similar histology and cell morphology to the patient tumor. Though few studies have focused on the genomic similarities of patient tissue and primary xenografts, those published suggest that mutations are generally conserved. $^{15,16}$ In addition, because these models are established under more physiological conditions (ie, temperature, oxygen levels, nutrient content etc.), they may be appropriate for representing human cancers in specific settings.

Some attempts have been made to establish models of primary E/GEJ cancer xenografts. For E/GEJ adenocarcinoma, previous studies have successfully established primary xenografts using nude mice. ${ }^{17,18}$ Similarly, xenografts have been created from various primary squamous cell carcinomas. ${ }^{19}$ These studies, however, have not focused on the factors that might contribute to engraftment. We sought to identify the clinicopathological and technical predictors of engraftment in PTXGs and to determine the representativeness of PTXGs to model human cancer biology, chemoresistance and their utility in testing novel therapeutics.

\section{MATERIALS AND METHODS}

\section{Animals}

NOD/SCID and NOD/SCID/IL2R $\gamma^{-1-}$ mice were bred internally at the OCI Animal Care facility and ranged from 4-6 weeks old. All animals were kept in a pathogen free environment on a standard 12-hr day/ 12-hr night cycle and were fed a standard sterilized pellet diet and water ad libitum. Animals were treated under the ethical guidelines of the Animal Care Committee at the OCI Animal Care Facility.

\section{Tissue Processing, Implantation and Treatment}

The study was approved by the Research Ethics Board at University Health Network. E/GEJ cancer tissue (known as P0 or Passage 0) was obtained from patients undergoing E/ GEJ resection at Toronto General Hospital, Toronto, ON. Biopsy specimens were obtained from patients undergoing endoscopies for pathological confirmation of carcinoma at Toronto General Hospital. In both cases, fresh tissue was stored in RPMI 1640 medium (no FBS added) until it was cut into small pieces of $\sim 5 \mathrm{~mm} \times 5 \mathrm{~mm}$ by using a sterile surgical blade. One representative piece was saved in Optimal Cutting Temperature (OCT) compound and frozen at $-80^{\circ} \mathrm{C}$ for further molecular analysis. Within less than $24 \mathrm{~h}$, pieces were implanted subcutaneously, initially in the lowerright abdomen, and later the flank of NOD/SCID mice. Mice were palpated regularly for signs of tumor growth. Once palpable, tumors were measured twice weekly until they had reached a maximum size of $1.5 \mathrm{~cm}$. When the tumor was surgically removed, representative pieces were preserved in various fixatives for additional analysis. H\&E slides were made from FFPE blocks in order to follow any changes in morphology and to confirm the epithelial nature of the tumor. The rest of the tumor was sectioned in $5 \mathrm{~mm} \times 5 \mathrm{~mm}$ pieces, which were reimplanted in a new generation of mice subcutaneously. This process was repeated for every passage. Tumors were considered engrafted if they were passaged at least once in vivo (ie, P1). Tumors were considered not to have engrafted if no tumor growth was detected for at least 6 months after implantation (ie, no P1) or if the mass was caused by a non-epithelial cell proliferation (eg, lymphoid cells only).

Chemosensitivity experiments were conducted on cohorts of 10 mice per group. Once tumors reached $\sim 300 \mathrm{~mm}^{3}$, mice were randomized into control and treatment arms. The chemotherapy group was treated with $5.4 \mathrm{mg} / \mathrm{kg}$ of cisplatin ( $1 \mathrm{mg} / \mathrm{ml}$, Hospira, DIN: 02126613) and $9 \mathrm{mg} / \mathrm{kg}$ of paclitaxel ( $2 \mathrm{mg} / \mathrm{ml}$, Hospira, DIN: 02296624), while the control group was treated with saline. Tumors were measured twice a week, using metric calipers. Volume was calculated using the ovoid formula: Volume $=\left(\text { Length }{ }^{\star} \text { Width }^{2}\right)^{\star} 0.52$.

\section{Short Tandem Repeat (STR) Analysis}

STR profiling was attempted on all xenograft lines. We compared profiles from patient tissues to the latest xenograft passage from each line. Ten slices from OCT frozen samples or FFPE blocks were cut, each $10-\mu \mathrm{m}$ thick, and DNA was extracted using the phenol-chloroform method. STR analysis interrogated 16 different loci, using the AmpFISTR Identifiler PCR kit (Applied Biosystems: 4322288).

\section{Clinical and Pathological Information}

History of Barrett's esophagus, heartburn, alcohol and smoking were obtained from self-administered patient questionnaires while treatment and outcome information (ie, overall survival (OS)) was obtained through the electronic patient records (EPR) system at PMH. Pathological data for each patient was reviewed and compared with each xenograft passage by a team of subspecialized pathologists.

\section{Statistical Analysis}

Engraftment was correlated to clinicopathologic features, and to OS. Fisher's exact and $t$-tests were used to determine the univariate association of individual clinicopathological features with engraftment. Multivariate logistic regression was used to identify independent predictors of engraftment. OS was defined as time between surgery and the last date known alive or date of death. Disease-free survival (DFS) was defined as the time between surgery and last date known without progression/recurrence/death or date of progression/ recurrence/death. The Kaplan-Meier method was used to calculate OS and the log-rank test was used to test significance. A Cox proportional hazards model was used to determine whether clinicopathological factors affected 
survival. Patient samples that were histologically negative for tumor at the time of resection (ie, the patient had a complete response after neo-adjuvant chemo-radiation) were analyzed separately. PTXGs derived from biopsy specimens were also analyzed separately. Two-sided tests were applied. Results were considered significant if the $P$-value was less than or equal to 0.05 . Statistical analyses were performed using SAS v.9.2 (Cary, NC).

\section{RESULTS}

\section{Engraftment}

A total of 96 patient tumors were implanted between April 2007 and September 2011. Ninety were from fully resected specimens while six were from tumor-derived biopsies. Of these, eight tumors were found to be rare tumor types such as melanoma, sarcoma and neuroendocrine neoplasms, (Figure 1) which were removed from further analysis. Two additional tumors were reclassified as gastric cancer and were also removed from the analysis. Of the remaining 80 tumors,
11 were separately analyzed because they came from patients who had a complete response to neo-adjuvant chemo-radiation and had no viable tumor cells in the pathological specimen at the time of resection (eg, pT0). Of these, one sample successfully engrafted and was included in the main engraftment analysis. In total, $70 \mathrm{E} / \mathrm{GEJ}$ cancers (16 squamous cell carcinomas and 54 adenocarcinomas) were analyzed for engraftment in NOD/SCID mice (Figure 1). Twenty-three tumors engrafted successfully: 18/54 (33\%) adenocarcinomas and 5/16 (31\%) squamous cell carcinomas. Two lymphoid proliferations without evidence of an epithelial tumor were found at passage one in two mice that had been implanted with adenocarcinomas; one additional lymphoid proliferation occurred in a mouse implanted with a squamous cell carcinoma in the second passage. A small subset of implantations was carried out in both NOD/SCID and NOD/SCID/IL2R $\gamma^{-1-}$ mice (in parallel) but no apparent difference in engraftment was found $(n=4)$. Pretreatment endoscopic biopsies had a $50 \%$ (3/6) engraftment rate.

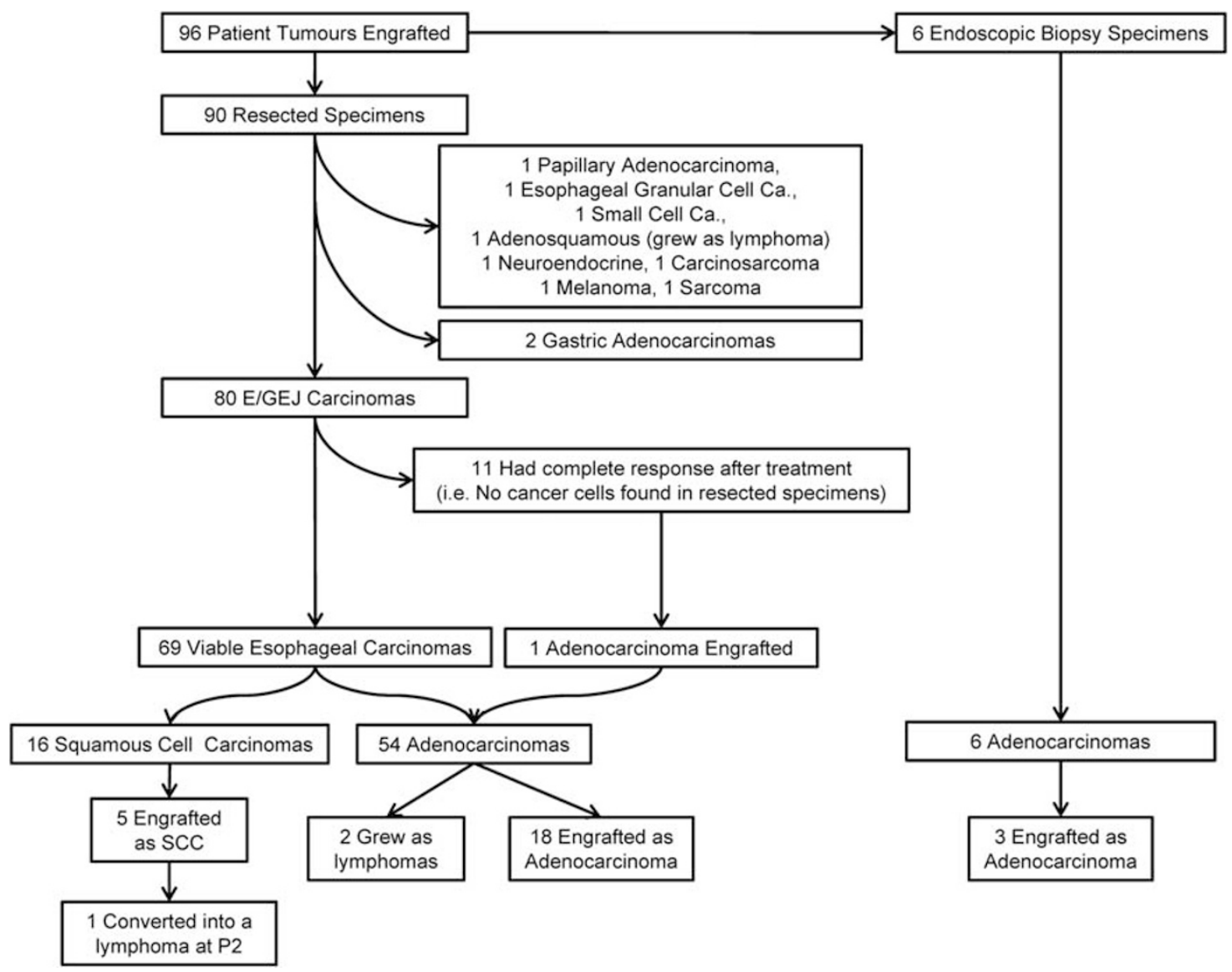

Figure 1 Schematic of the total number of implantations conducted during a span of 4 years. In total, 96 implantations were carried out, six of which were endoscopic biopsies. Eight were of rare histological types while two were reclassified as gastric cancers. Of the remaining 80 tumors 11 were found to have no viable cells in the clinical specimen (after treatment); One of these specimens engrafted and was reintroduced into the pool of implanted tumors. Of the implanted tissues 54 were adenocarcinomas and 16 were squamous cell carcinomas. From the adenocarcinomas, 18 were successfully engrafted as E/GEJ cancers while two grew as lymphomas. In the squamous cell carcinoma pool, five were engrafted and one converted into a lymphoma after the second passage. Of the six endoscopic biopsies that were implanted, three were engrafted successfully. 
Table 1 Patient demographics for implanted esophageal cancer tumors

\begin{tabular}{|c|c|c|c|c|}
\hline Characteristics & $\begin{array}{l}\text { Adenocarcinoma } \\
\qquad(n=54)\end{array}$ & $\begin{array}{l}\text { Squamous cell } \\
\text { carcinoma } \\
(n=16)\end{array}$ & $P$-value ${ }^{a}$ & $\begin{array}{c}\text { All } \\
\text { tumors } \\
(n=70)\end{array}$ \\
\hline Mean age (range) & $63(44-87)$ & $66(46-85)$ & $0.45^{\mathrm{b}}$ & $\begin{array}{c}63.7 \\
(43.8-86.5)\end{array}$ \\
\hline $\begin{array}{l}\text { Disease-free } \\
\text { survival time }\end{array}$ & & & & \\
\hline $\begin{array}{l}\text { Median (years) } \\
\text { Sex }\end{array}$ & 1.4 & DNR & $0.28^{c}$ & 1.45 \\
\hline Male & $41(76 \%)$ & 11 (69\%) & 0.54 & $52(74 \%)$ \\
\hline Female & $13(24 \%)$ & $5(31 \%)$ & & $18(26 \%)$ \\
\hline
\end{tabular}

Pathological stage
$\begin{array}{llrll}1-2 & 19(35 \%) & 11(69 \%) & 0.02 & 30(43 \%) \\ 3-4 & 35(65 \%) & 5(31 \%) & & 40(57 \%)\end{array}$

Differentiation $^{\text {d }}$

$\begin{array}{lrrrr}\text { Poor } & 22(41 \%) & 3(19 \%) & 0.22 & 25(36 \%) \\ \text { Moderate/well } & 29(54 \%) & 11(69 \%) & & 39(56 \%)\end{array}$

Location of malignancy

\begin{tabular}{|c|c|c|c|c|}
\hline Upper esophagus & 0 & $1(6 \%)$ & & $1(1 \%)$ \\
\hline Middle esophagus & $2(4 \%)$ & $7(44 \%)$ & $<0.001$ & $9(13 \%)$ \\
\hline Lower esophagus & $10(19 \%)$ & $6(38 \%)$ & & $16(23 \%)$ \\
\hline GEI & $42(78 \%)$ & $2(13 \%)$ & & $44(630$ \\
\hline
\end{tabular}

Neo-adjuvant chemo-rad

$\begin{array}{lrrrr}\text { Yes } & 24(44 \%) & 5(31 \%) & 0.51 & 29(41 \%) \\ \text { No } & 30(56 \%) & 11(69 \%) & 41(59 \%)\end{array}$

History of heartburn

$\begin{array}{lllll}\text { Yes } & 26(48 \%) & \text { N/A } & \text { N/A } & \text { N/A } \\ \text { No } & 28(52 \%) & & & \end{array}$

Barrett's esophagus

$\begin{array}{lllll}\text { Yes } & 13(24 \%) & \text { N/A } & \text { N/A } & \text { N/A } \\ \text { No } & 41(76 \%) & & & \end{array}$

Smoking status

$\begin{array}{lcccc}\text { Current smoker } & 5(9 \%) & 2(13 \%) & & 7(10 \%) \\ \text { Ex-smoker } & 30(56 \%) & 9(56 \%) & 0.9 & 39(56 \%) \\ \text { Never smoker } & 19(35 \%) & 5(31 \%) & & 24(34 \%)\end{array}$

Table 1 Continued

\begin{tabular}{|c|c|c|c|c|}
\hline Characteristics & $\begin{array}{c}\text { Adenocarcinoma } \\
\qquad(n=54)\end{array}$ & $\begin{array}{l}\text { Squamous cell } \\
\text { carcinoma } \\
(n=16)\end{array}$ & $P$-value ${ }^{\mathrm{a}}$ & $\begin{array}{c}\text { All } \\
\text { tumors } \\
(n=70)\end{array}$ \\
\hline \multicolumn{5}{|c|}{ Alcohol consumption $^{\mathrm{d}}$} \\
\hline Current drinker & & $7(44 \%)$ & & \\
\hline Ex-drinker & $\mathrm{N} / \mathrm{A}$ & $2(13 \%)$ & $\mathrm{N} / \mathrm{A}$ & $\mathrm{N} / \mathrm{A}$ \\
\hline Never drinker & & $2(13 \%)$ & & \\
\hline
\end{tabular}

Abbreviations: GEJ, gastro-esophageal junction; N/A, not applicable.

${ }^{\text {a}}$ Fisher's exact test unless otherwise stated; comparing adenocarcinoma and squamous cell carcinoma.

${ }^{\mathrm{b}}$ Student's $t$-test.

'Log-rank test.

dPatients with no available information were not included in the calculation.

\section{Patient Demographics}

Demographics were analyzed for the 70 patients with viable resected E/GEJ cancer, including factors such as DFS, pathological stage, differentiation, location of malignancy and preoperative treatment (Table 1). Only $31 \%$ of patients with squamous cell carcinomas presented with advanced stage disease compared with $65 \%$ of patients with adenocarcinoma $(P=0.02)$. As expected, the vast majority of adenocarcinomas were found in the GEJ (78\%) while squamous cell carcinomas were more prevalent in the mid-esophagus (44\%, $P<0.001)$. Age, DFS, differentiation and neo-adjuvant chemo-radiation were not significantly different between the two subtypes.

\section{Clinicopathological Predictors of Engraftment}

A summary of all clinicophathological characteristics is shown in Table 2. Each characteristic was first analyzed in all tumors and separated by histology: adenocarcinomas and squamous cell carcinomas.

Overall, tumors that had been pretreated with chemoradiation had a lower chance of engraftment $(P=0.02$, Table 3). Changing the implantation site from the lower-right abdomen to the flank significantly improved our engraftment rate by twofold $(P=0.04)$. In a multivariate analysis, only neo-adjuvant chemo-radiation remained significantly associated with a lower chance of engraftment (OR: 0.27 , CI: 0.09-0.84).

When evaluating adenocarcinomas alone, (Table 3), the only significant univariate factors that correlated with improved engraftment were poor differentiation $(P=0.04)$ and lack of neo-adjuvant treatment with chemo-radiation $(P=0.03)$. Because of their etiological association with E/GEJ adenocarcinoma, gastro-esophageal reflux disease and Barrett's esophagus were also evaluated as predictors of engraftment; no significance was found $(P>0.05$, each comparison). Multivariate analysis showed that only poor 
Table 2 Clinical and pathological features of implanted tumors

\begin{tabular}{|c|c|c|c|c|c|c|}
\hline \multirow{2}{*}{ Characteristics } & Engrafted & Non-engrafted & Engrafted & Non-Engrafted & Engrafted & Non-Engrafted \\
\hline & $(n=23)$ & $(n=47)$ & $(n=18)$ & $(n=36)$ & $(n=5)$ & $(n=11)$ \\
\hline \multicolumn{7}{|c|}{ Disease-free survival time } \\
\hline Median (years) & 1.39 & 1.86 & 1.39 & 1.45 & DNR & DNR \\
\hline \multicolumn{7}{|l|}{ Sex } \\
\hline Male & $15(65 \%)$ & 37 (79\%) & $14(78 \%)$ & $27(75 \%)$ & $1(20 \%)$ & $10(91 \%)$ \\
\hline Female & 8 (35\%) & $10(21 \%)$ & $4(22 \%)$ & $9(25 \%)$ & $4(80 \%)$ & 1 (9\%) \\
\hline $3-4$ & $16(70 \%)$ & $24(51 \%)$ & $14(78 \%)$ & $21(58 \%)$ & $2(40 \%)$ & $3(27 \%)$ \\
\hline \multicolumn{7}{|l|}{ Differentiation } \\
\hline Poor & $12(52 \%)$ & $13(28 \%)$ & $11(61 \%)$ & $11(31 \%)$ & $1(20 \%)$ & $2(18 \%)$ \\
\hline Moderate/well & $10(44 \%)$ & $30(64 \%)$ & $6(33 \%)$ & $23(64 \%)$ & $4(80 \%)$ & 7 (64\%) \\
\hline \multicolumn{7}{|l|}{ Location of malignancy } \\
\hline Upper esophagus & $1(4 \%)$ & $0(0 \%)$ & & & $1(20 \%)$ & 0 \\
\hline Middle esophagus & $3(13 \%)$ & $6(13 \%)$ & $0(0 \%)$ & $2(6 \%)$ & $3(60 \%)$ & $4(36 \%)$ \\
\hline Lower esophagus & $5(22 \%)$ & $11(23 \%)$ & $4(22 \%)$ & $6(17 \%)$ & $1(20 \%)$ & $5(46 \%)$ \\
\hline GEJ & $14(61 \%)$ & $30(64 \%)$ & $14(78 \%)$ & $28((78 \%)$ & 0 & $2(18 \%)$ \\
\hline \multicolumn{7}{|l|}{ History of heartburn } \\
\hline No & & & $9(50 \%)$ & 19 (53\%) & & \\
\hline \multicolumn{7}{|l|}{ Barrett's esophagus } \\
\hline Yes & N/A & N/A & $2(11 \%)$ & $11(31 \%)$ & N/A & N/A \\
\hline No & & & $16(89 \%)$ & $25(69 \%)$ & & \\
\hline \multicolumn{7}{|l|}{ Smoking status } \\
\hline Current smoker & & & $1(6 \%)$ & $4(11 \%)$ & 0 & $2(18 \%)$ \\
\hline Ex-smoker & $\mathrm{N} / \mathrm{A}$ & N/A & $13(72 \%)$ & $17(47 \%)$ & $4(80 \%)$ & $5(46 \%)$ \\
\hline Never smoker & & & $4(22 \%)$ & $15(42 \%)$ & $1(20 \%)$ & $4(36 \%)$ \\
\hline \multicolumn{7}{|l|}{ Alcohol consumption } \\
\hline Current drinker & & & & & $2(40 \%)$ & $5(46)$ \\
\hline Ex-drinker & N/A & N/A & N/A & $\mathrm{N} / \mathrm{A}$ & 0 & $2(18 \%)$ \\
\hline Never drinker & & & & & $1(20 \%)$ & $1(9 \%)$ \\
\hline \multicolumn{7}{|l|}{ Site of implantation } \\
\hline Abdomen & 7 (30\%) & $28(60 \%)$ & 7 (39\%) & $20(56 \%)$ & 0 & $8(73 \%)$ \\
\hline Flank & $16(70 \%)$ & 19 (40\%) & $11(61 \%)$ & $16(44 \%)$ & 5 (100\%) & $3(27 \%)$ \\
\hline
\end{tabular}

Abbreviations: GEJ, gastro-esophageal junction; N/A, not applicable. 
Table 3 Significant clinicopathological characteristics of engraftment

\begin{tabular}{lcccc}
\hline Characteristics & $\begin{array}{c}\text { Engrafted } \\
\text { no. of }\end{array}$ & $\begin{array}{c}\text { Non-engrafted } \\
\text { no. of patients }\end{array}$ & $\begin{array}{c}\text { Odds } \\
\text { ratio }\end{array}$ & $P$-value \\
patients (\%) & $(\%)$ & $(95 \% \mathrm{Cl})$ &
\end{tabular}

\begin{tabular}{|c|c|c|c|c|}
\hline \multicolumn{5}{|c|}{ All subtypes $(n=70)$} \\
\hline \multicolumn{5}{|c|}{ Neo-adjuvant chemo-rad } \\
\hline Yes & $5(22 \%)$ & $24(51 \%)$ & 0.27 & $0.02^{\mathrm{a}}$ \\
\hline No & $18(78 \%)$ & $23(49 \%)$ & $(0.1-0.8)$ & \\
\hline \multicolumn{5}{|c|}{ Site of implantation } \\
\hline Abdomen & $7(30 \%)$ & $28(60 \%)$ & 0.3 & 0.04 \\
\hline Flank & $16(70 \%)$ & 19 (40\%) & $(0.1-0.7)$ & \\
\hline
\end{tabular}

\begin{tabular}{|c|c|c|c|c|}
\hline \multicolumn{5}{|c|}{ Adenocarcinoma $(n=54)$} \\
\hline \multicolumn{5}{|l|}{ Differentiation $^{\text {b }}$} \\
\hline Poor & $11(61 \%)$ & $11(31 \%)$ & 3.67 & \multirow[t]{2}{*}{$0.04^{\mathrm{a}}$} \\
\hline Moderate/well & $6(33 \%)$ & $23(64 \%)$ & $(1.1-12.6)$ & \\
\hline \multicolumn{5}{|c|}{ Neo-adjuvant chemo-rad } \\
\hline Yes & $4(22 \%)$ & $20(56 \%)$ & 0.23 & \multirow[t]{2}{*}{0.03} \\
\hline No & $14(78 \%)$ & $16(44 \%)$ & $(0.1-0.8)$ & \\
\hline \multicolumn{5}{|c|}{ Squamous cell carcinoma $(n=16)$} \\
\hline Mean age (range) & $74.3(61-85)$ & $61.5(46-73)$ & $1.21(1.0-1.6)$ & 0.02 \\
\hline \multicolumn{5}{|l|}{ Sex } \\
\hline Male & $1(20 \%)$ & $10(91 \%)$ & 0.03 & \multirow[t]{2}{*}{$0.02^{\mathrm{a}}$} \\
\hline Female & $4(80 \%)$ & $1(9 \%)$ & $(0.001-0.5)$ & \\
\hline \multicolumn{5}{|l|}{ Site of implantation } \\
\hline Abdomen & 0 & $8(73 \%)$ & 0.04 & \multirow[t]{2}{*}{0.03} \\
\hline Flank & $5(100 \%)$ & $3(27 \%)$ & $(0.002-0.9)$ & \\
\hline
\end{tabular}

Abbreviation: $\mathrm{Cl}$, confidence interval.

Fisher's exact test was used to compare engrafted and non-engrafted predictors.

Student's $t$-test was used for to determine if age was a predictor of engraftment.

${ }^{a}$ Only significant variable in multivariate analysis.

${ }^{b}$ Patients with no available information on differentiation were not included in the calculation.

differentiation was significantly associated with the ability to engraft (OR: 3.67, CI: 1.07-12.55).

Because there were few squamous cell carcinoma cases in this study, analyses of predictors were considered exploratory. Nonetheless, in this subset, older individuals $(P=0.02)$ and women $(P=0.02)$ had a significantly higher chance of engraftment upon univariate analysis (Table 3 ). Factors such as smoking status and alcohol consumption were not significantly associated with engraftment. Implanting samples subcutaneously in the flank proved to have significantly higher engraftment than implanting them subcutaneously in the abdomen $(P=0.03)$. In an exploratory multivariate analysis, being female was the only variable that remained significantly associated with improved engraftment (OR: 0.03, CI: 0.001-0.5, $P=0.02$ ).

In evaluating all engrafted tumors, time to second passage (time from P0 implantation until the end of P1 and passaging to P2) was tested as a potential surrogate marker of tumor aggressiveness whereby more aggressive tumors might engraft faster and grow faster. ${ }^{9}$ Time to second passage was compared with many potential predictors, including histological differentiation, stage and neoadjuvant chemo-radiation status. Poor differentiation was the only factor that showed a univariate trend towards shorter time to second passage $(P=0.07)$; no associations were significant.

\section{Pathological Characteristics of Xenografts}

Using our panel of xenograft models, we demonstrated, through blind assessment by light microscopy, that tissue morphology remained similar between passages, correlating well with the original patient tumor (Figures $2 \mathrm{a}-\mathrm{b}, \mathrm{d}-\mathrm{e}, \mathrm{g}-\mathrm{h}$ ). Once engrafted, histological features were similar through serial passaging (Figures $2 \mathrm{~b}-\mathrm{c}, \mathrm{e}-\mathrm{f}, \mathrm{h}-\mathrm{i}$ and $\mathrm{k}-\mathrm{l}$ ), even in late passages such as P10 (Figures $2 \mathrm{~b}-\mathrm{c}$ ). Some tumors showed more conspicuous morphological differences between patient and xenograft (Figures 2j-1), but reflected variability in sampling, as there were always specific areas of the tumor that matched the morphology and differentiation grade of the xenograft (Supplementary Tables 3 and 4). Morphologic heterogeneity observed in the original tumor (Figures $3 \mathrm{a}$ and b) was also reflected in xenografts of the first passage (Figures $3 \mathrm{c}-\mathrm{d})$ and was mostly conserved after serial passaging (Figures $3 \mathrm{e}-\mathrm{f}$ ). Although the vast majority of xenografts remained similar across passages, a small minority exhibited minor changes such as differentiation into a signet subtype (one adenocarcinoma) and loss of keratinization (one squamous cell carcinoma). Generally, xenografts exhibited a higher amount of mitotic cells when compared with their P0 counterparts. Overall, case-by-case assessment found that stroma did not noticeably change with serial passaging.

Consistent with other studies, ${ }^{9,20}$ we have also seen the formation of lymphoid proliferations/lymphomas in four implantations. Three cases (one adenosquamous and two adenocarcinomas) were replaced by tumor-forming lymphoid proliferations in P1. Another case was seen with an established squamous cell xenograft that was replaced by a lymphoid mass in passages 2 and 3.

None of the tumors exhibited metastasis from the initial site of implantation and all initially implanted tissue was able to reach late passages (unless discontinued for technical reasons). A full list of characteristics for all engrafted and non-engrafted tumors is presented in Supplementary Tables 1 and 2, respectively.

STR profiles of 18 samples closely matched their corresponding patient STR. One differed across many loci, 

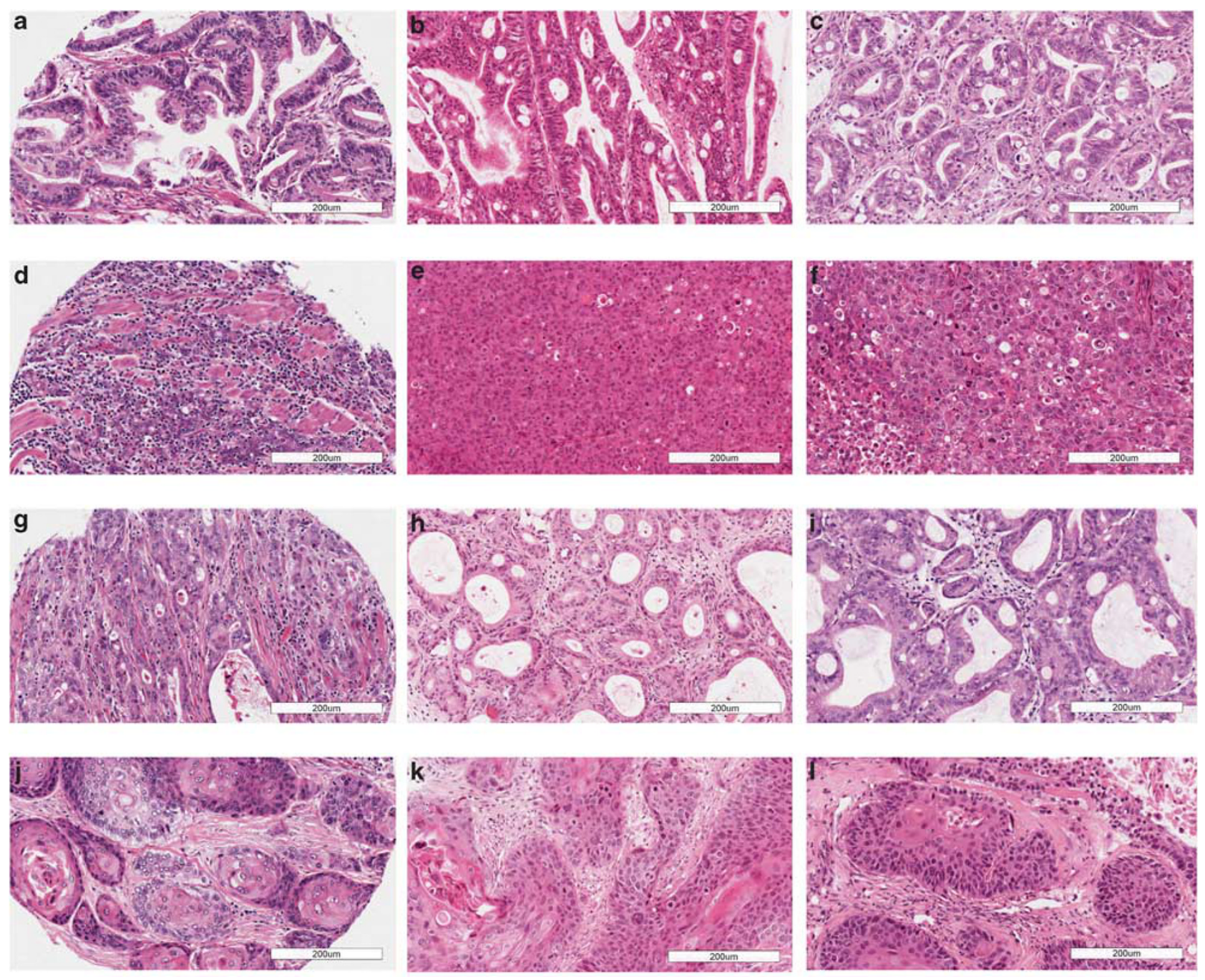

Figure 2 The histology of PTXGs is maintained through serial passaging. (a) A moderately differentiated adenocarcinoma (P0, patient) maintains a similar morphology with identifiable gland formation in the xenografts of (b) passage 1 through to (c) passage 10. (d) A poorly differentiated adenocarcinoma with solid tumor pattern (P0) maintains its morphologic features in (e) passage 1 to (f) passage 4. (g) Moderately/poorly differentiated adenocarcinoma from patient (P0) shows (h) a more moderately differentiated histology in passage 1 and (i) passage 3 but still retains similar features. (j) A well/moderately differentiated squamous cell carcinoma with numerous squamous 'nests' (PO) shows similar morphology in (k) passage 1 to (I) passage 3.

exhibiting change across passages; this represented underlying genetic instabilities, and not line contamination as the progression of instability could be traced from passage to passage. The four remaining samples could not be compared due to lack of available, high quality, non-degraded research tissue. Even though these samples could not be confirmed through STR, their morphology and differentiation closely matched that of the original patient.

\section{Ability to Engraft and Survival}

To determine if engrafted tumors were more aggressive than their non-engrafted counterparts, we assessed the OS and DFS for patients by engraftment status. In addition, to eliminate the effects of neo-adjuvant chemo-radiation, we performed the same analysis in the subgroup of patients that was not treated with chemo-radiation.

The 70 resected patients had a median OS of 2.17 years and median DFS of 1.45 years; no significant differences by engraftment status were observed. The hazard ratios for OS by engraftment status (engraftment $v s$ non-engraftment) are presented in Supplementary Figure 1A. The hazard ratios for DFS were 1.2 (CI: 0.5-3.0) for all patients and 1.02 (CI:0.4-2.5) for the adenocarcinoma subset. Results were similarly negative after neo-adjuvant chemo-radiation status was taken into account (Supplementary Figure 1B, $P>0.2$ ). 

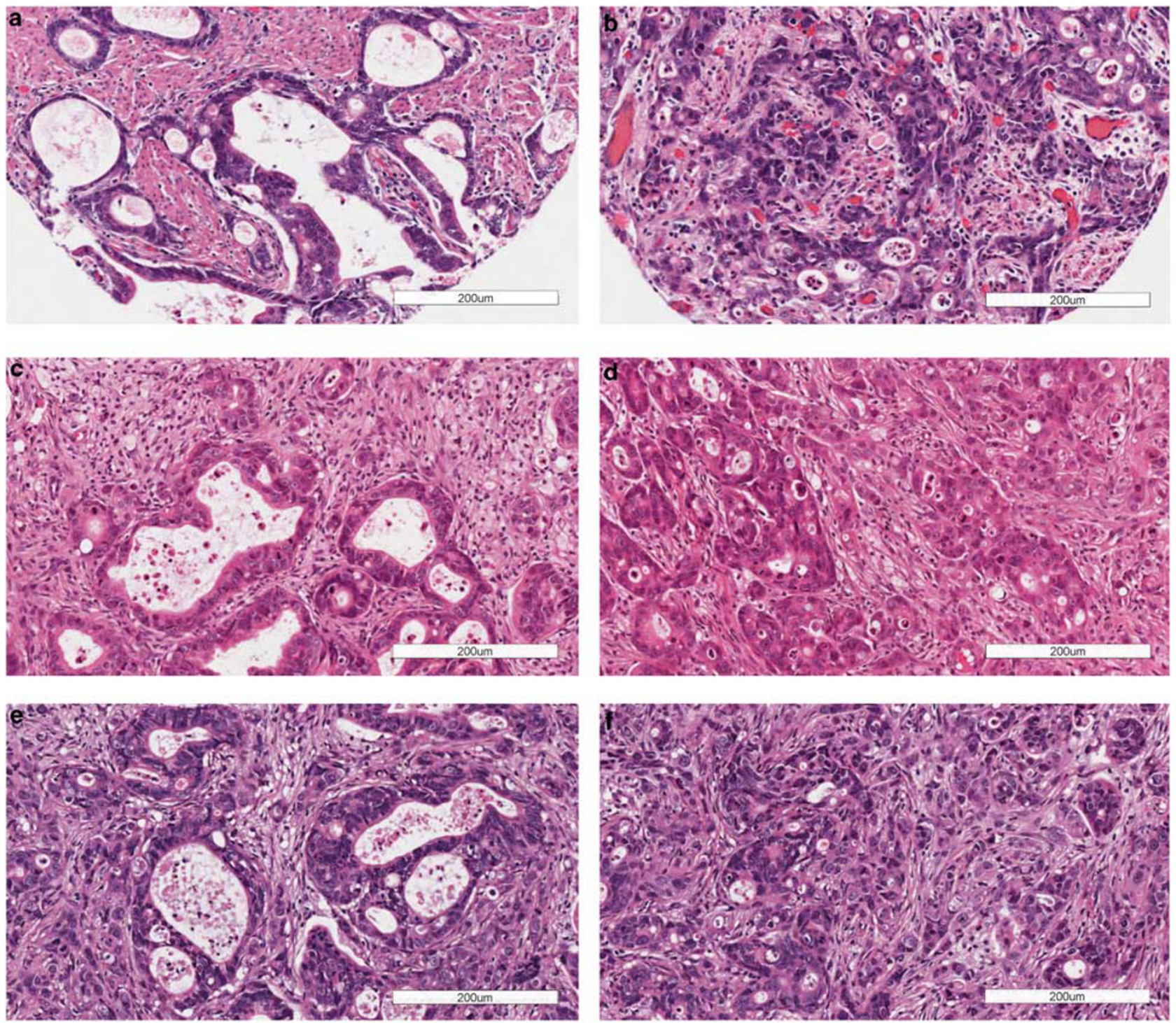

Figure 3 The morphologic heterogeneity of the primary tumor is represented in corresponding xenografts. Areas with well/moderate differentiation (a) are acompanied by areas of poorer differentiation as indicated by a less obvious gland formation (b) within the patient tumor. Similarly, the P1 xenograft derived from this patient shows a similar spectrum of differentiation from well/moderate (c) to poor (d) within a single xenograft tumor. (e) and (f) shows this heterogeneity at passage 3.

\section{Chemosensitivity of E/GEJ Cancer Xenografts}

Chemosensitivity of two PTXG models is presented in Figure 4 to determine the utility of such models for studying new therapies and therapy resistance. Each line was treated with a combined single dose of cisplatin $(5.4 \mathrm{mg} / \mathrm{kg})$ and paclitaxel $(9 \mathrm{mg} / \mathrm{kg})$ when the average tumor size was in between $250-300 \mathrm{~mm}^{3}$. Each treatment arm had 10 mice for a total of 20 mice tested for each PTXG model. Line one was treated on the 55th day after implantation and exhibits a clear growth delay indicating that it is chemosensitive (Figure 4a). In contrast, line eight was treated on day 30 and showed no delay in growth compared with the control (Figure 4b).

\section{DISCUSSION}

In this study, we have confirmed the feasibility of creating primary human E/GEJ cancer xenograft models using freshly resected and endoscopic biopsy-derived tumor specimens from patients. Contrary to previous studies, ${ }^{17-19}$ we focus less on feasibility and more on whether any clinical parameters correlated with engraftment as well as how representative the xenografts are of patient samples. In studying the characteristics of engrafted samples we hope to create a preclinical model that can be used to investigate carcinogenesis, identify mechanisms of resistance to current treatments and test new therapeutic agents.

The highest proportion of engrafted samples was seen in patients whose resected tumors were not treated with 
a

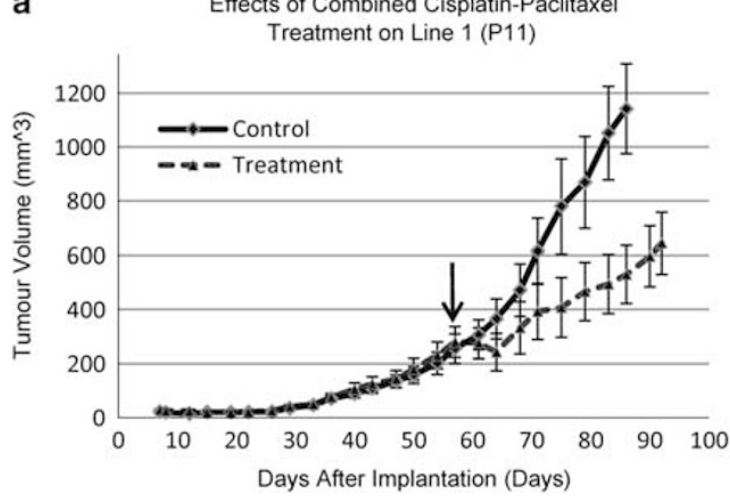

b

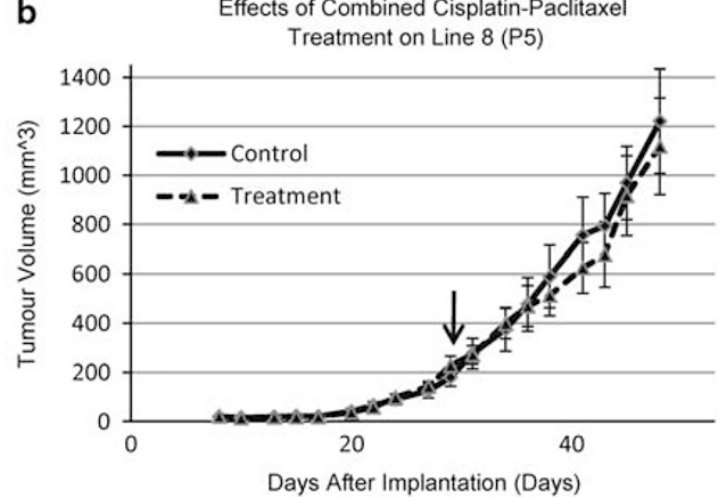

Figure 4 Chemosensitivity of xenograft lines to cisplatin and paclitaxel. Xenograft lines received a combined dose of cisplatin and paclitaxel when the tumors averaged $250 \mathrm{~mm}^{3}$ to $300 \mathrm{~mm}^{3}$ (black arrow indicates time of treatment). (a) Line 1 was treated in passage 11 and was chemo-sensitive while (b) line 8 was treated in passage 5 and proved to be chemo-resistant.

neo-adjuvant chemo-radiation (44\%). Although the rate decreased significantly $(17 \%)$ in patients treated with neoadjuvant chemo-radiation, some tumors did engraft. Samples that engrafted despite being pre-treated with chemo-radiation are important because they may represent models of resistance. Engraftment rates were lowest in the subset of patients that had no viable tumor left after treatment (9\%). As a result of our experiments with resected specimens, we started to implant treatment-naive endoscopic biopsies to improve the representativeness of these models for studying carcinogenesis and molecular biology. We demonstrated a similar engraftment rate (50\%) compared with untreated resection tissue. Thus the feasibility of using endoscopic biopsies to create xenograft models greatly increases the utility and scope of our PTXG models. PTXGs may be potentially useful for testing sensitivity of therapeutic agents commonly used in clinic: we discovered a PTXG model sensitive to cisplatin-paclitaxel chemotherapy (Line 1) and another model that was resistant (line 8). The potential usefulness of these models requires additional research.

Consistent with other studies, ${ }^{8,20}$ we found lymphoid proliferation in a small group of mice $(3 / 80$ or $4 \%)$. These lymphomas might come from mouse origin as no visible signs of lymphoid growth were apparent in the original tumor or early passage xenografts. In any case, this indicates a potential limitation to this model as unwanted lymphoid growth might interfere with normal experiments.

Previous models of E adenocarcinoma that were developed using nude mice ${ }^{17,18}$ showed engraftment rates of $\sim 30-35 \%$. None of these studies indicated whether their samples received any neo-adjuvant chemo-radiation. If these previous studies involved primarily untreated patient tissue, then our PTXG models represent a 10-15\% improvement in the engraftment rate. Similarly, squamous cell carcinoma xenografts have also been established using nude mice ${ }^{19}$ and showed an engraftment rate of $\sim 44 \%$. Again, there was no indication whether samples received neo-adjuvant chemo- radiation. By using the same implantation procedures, we also demonstrated that adenocarcinomas and squamous cell carcinomas have similar engraftment rates.

In the E/GEJ adenocarcinomas, poor differentiation was the only significant factor associated with improved engraftment in multivariate analysis. Poorly differentiated tumors have higher engraftment rates in other xenograft models such as early stage lung, ${ }^{9}$ head and neck, ${ }^{21}$ and breast cancer. ${ }^{10}$ All of these models also showed a lower overall patient survival for engrafted specimens when compared with their non-engrafted counterparts, suggesting a bias towards engraftment of more aggressive tumors. In contrast to these other models, however, there was little indication that engrafted E/GEJ tumors in our sample were associated with more aggressive tumor behavior than non-engrafted models, even after accounting for neo-adjuvant chemo-radiation in the analysis. The only evidence was a non-significant trend towards early passaging in poorly differentiated tumors at P1. This suggests, but does not prove, that our E/GEJ PTXG models may be less biased towards engraftment of only the most aggressive forms of these cancers. Unlike these other cancer sites, E/GEJ cancers are generally very aggressive; our patient sample had a median DFS of only 1.4 years. The uniform aggressiveness of these tumors might explain why engrafted and non-engrafted tumors have similar OS even after adjusting for clinical factors such as stage and differentiation.

Only 5 of 16 squamous cell carcinomas engrafted. The small denominator represents the North American trend towards a decreasing incidence of squamous cell carcinomas, but poses significant limitations to our understanding of the predictors of engraftment in these samples. Although the multivariate analysis suggested that tumors from women engrafted at a higher rate, the univarate analysis suggests that, in addition, older patients and those engrafted in the flank were also more likely to engraft. A larger sample is necessary to draw conclusions. 
When we changed the location of implantation from the abdomen to the flank of the mice half way through this series, engraftment rates improved. This alteration may have resulted in a change of microenvironment. In contrast, implantations in the mouse esophagus (the orthotopic site) were not performed because such tumors may obstruct food from entering the stomach, causing the mice to starve and render serial tumor measurements more difficult.

The majority of engrafted PTXGs histologically resembled their parent tumor (P0) and remained morphologically stable after multiple passages. Within some P0 tumors, heterogeneity of tissue morphology was observed, where some tumor areas were poorly differentiated while other areas were moderately differentiated. Similar heterogeneity was seen in the corresponding xenograft, a phenomenon not typically described in cell lines. Unlike xenograft models of ovarian cancer $^{22}$ and adenoid cyst carcinoma, ${ }^{23}$ the histology and morphology of most E/GEJ PTXG models remained relatively stable even up to late passages (P5 and beyond). Beyond these late passages areas of necrosis and cellular debris notably increased. Genetically, most xenografts were stable across many passages. One exception was observed when considerable mutations appeared in later passages. This, however, is probably an indication of the genetic instability present in the patient tumor. In addition, no xenografts ceased to be passaged once established. Previous E adenocarcinoma ${ }^{17}$ and squamous cell carcinoma ${ }^{19}$ xenograft models reported that long-term multiply-passaged xenografts could only be established in $32 \%$ and $24 \%$ of all engrafted tumors, respectively. In our series, if a xenograft line did cease to be passaged in our series, it was due to loss of the mice from sickness or other complications. Even after accounting for losses due to any factor, we were still able to serially passage xenografts in greater than $80 \%$ of cases. Such a phenomenon might be related to a difference in the mouse strain: we utilized NOD/SCID mice compared with nude mice which are less immunocompromised. The full repertoire of immune cells is deficient in various degrees in all xenograft models. How the extent of immunosuppression affects the biology of the xenograft and its impact on various treatments is yet to be determined.

In conclusion, primary xenografts in NOD/SCID mice have the ability to reconstitute tumors that are morphologically similar to the patient tumor, demonstrating comparable heterogeneity. Although poor differentiation and lack of neo-adjuvant chemo-radiation were associated with improved engraftment rates, all disease stages and histological differentiations were engraftable, suggesting that creating a representative distribution of E/GEJ PTXGs is feasible. Endoscopic biopsies were also shown to be feasible in establishing PTXGs. Overall, this model has the potential to be used as a preclinical tool to study tumor biology, acquired and de novo resistance to therapies as well as to test sensitivity of novel therapeutics. In order to improve on our knowledge of these models, in the future, we will focus on their genetic and molecular stability.

Supplementary Information accompanies the paper on the Laboratory Investigation website (http://www.laboratoryinvestigation.org)

\section{ACKNOWLEDGEMENTS}

Funding: Alan B. Brown Chair in Molecular Genomics, Posluns Family Fund, CCO Chair in Experimental Therapeutics and Population Studies.

\section{DISCLOSURE/CONFLICT OF INTEREST}

The authors declare no conflict of interest.

1. Kim T, Grobmyer SR, Smith R, et al. Esophageal cancer-the five year survivors. J Surg Oncol 2011;103:179-183.

2. Alley MC, Scudiero DA, Monks A, et al. Feasibility of drug screening with panels of human tumor cell lines using a microculture tetrazolium assay. Cancer Res 1988;48:589-601.

3. Monks A, Scudiero D, Skehan P, et al. Feasibility of a high-flux anticancer drug screen using a diverse panel of cultured human tumor cell lines. J Natl Cancer Inst 1991;83:757-766.

4. Auman JT, McLeod HL. Colorectal cancer cell lines lack the molecular heterogeneity of clinical colorectal tumors. Clin Colorectal Cancer 2010;9:40-47.

5. Daniel VC, Marchionni L, Hierman JS, et al. A primary xenograft model of small-cell lung cancer reveals irreversible changes in gene expression imposed by culture in vitro. Cancer Res 2009;69: 3364-3373.

6. Ertel A, Verghese A, Byers SW, et al. Pathway-specific differences between tumor cell lines and normal and tumor tissue cells. Mol Cancer 2006;5:55.

7. Jones S, Chen WD, Parmigiani G, et al. Comparative lesion sequencing provides insights into tumor evolution. Proc Natl Acad Sci USA 2008;105:4283-4288.

8. Boonstra JJ, van Marion R, Beer DG, et al. Verification and unmasking of widely used human esophageal adenocarcinoma cell lines. J Natl Cancer Inst 2010;102:271-274.

9. John $T$, Kohler D, Pintilie $M$, et al. The ability to form primary tumor xenografts is predictive of increased risk of disease recurrence in early-stage non-small cell lung cancer. Clin Cancer Res 2011;17: 134-141.

10. Marangoni E, Vincent-Salomon A, Auger N, et al. A new model of patient tumor-derived breast cancer xenografts for preclinical assays. Clin Cancer Res 2007;13:3989-3998.

11. Rubio-Viqueira B, Jimeno A, Cusatis $G$, et al. An in vivo platform for translational drug development in pancreatic cancer. Clin Cancer Res 2006;12:4652-4661.

12. Fichtner I, Slisow W, Gill J, et al. Anticancer drug response and expression of molecular markers in early-passage xenotransplanted colon carcinomas. Eur J Cancer 2004;40:298-307.

13. Chen J, Milo GE, Shuler CF, et al. Xenograft growth and histodifferentiation of squamous cell carcinomas of the pharynx and larynx. Oral Surg Oral Med Oral Pathol Oral Radiol Endod 1996;81: 197-202.

14. Fujii E, Suzuki M, Matsubara K, et al. Establishment and characterization of in vivo human tumor models in the NOD/SCID/ gamma(c)(null) mouse. Pathol Int 2008;58:559-567.

15. Jeuken JW, Sprenger $\mathrm{SH}$, Wesseling $\mathrm{P}$, et al. Genetic reflection of glioblastoma biopsy material in xenografts: characterization of 11 glioblastoma xenograft lines by comparative genomic hybridization. J Neurosurg 2000;92:652-658.

16. Reyal F, Guyader C, Decraene C, et al. Molecular profiling of patient-derived breast cancer xenografts. Breast Cancer Res 2012; 14:R11.

17. de Both NJ, Wijnhoven BP, Sleddens HF, et al. Establishment of cell lines from adenocarcinomas of the esophagus and gastric cardia growing in vivo and in vitro. Virchows Arch 2001;438: 451-456. 
18. El-Rifai W, Harper JC, Cummings OW, et al. Consistent genetic alterations in xenografts of proximal stomach and gastroesophageal junction adenocarcinomas. Cancer Res 1998;58:34-37.

19. Kitamura $M$, Suda $M$, Nishihira $T$, et al. Heterotransplantation of human esophageal carcinoma to nude mice. Tohoku J Exp Med 1981; 135:259-264.

20. John T, Yanagawa N, Kohler D, et al. Characterization of lymphomas developing in immunodeficient mice implanted with primary human non-small cell lung cancer. J Thorac Oncol 2012;7:1101-1108.
21. Joshua B, Kaplan MJ, Doweck I, et al. Frequency of cells expressing CD44, a head and neck cancer stem cell marker: correlation with tumor aggressiveness. Head Neck 2012;34:42-49.

22. Stewart JM, Shaw PA, Gedye C, et al. Phenotypic heterogeneity and instability of human ovarian tumor-initiating cells. Proc Natl Acad Sci USA 2011;108:6468-6473.

23. Hashitani S, Noguchi K, Manno Y, et al. Changes of histological and biological features by serial passages in a human adenoid cystic carcinoma line transplantable in nude mice. Oncol Rep 2005;13:607-612. 